\title{
CIENCIAMATRIA
}

Revista Interdisciplinaria de Humanidades, Educación, Ciencia y Tecnología

Año VII. Vol. VII. N¹2. Enero - Junio. 2021

Hecho el depósito de ley: pp201602FA4721

ISSN-L: 2542-3029; ISSN: 2610-802X

Universidad Nacional Experimental Francisco de Miranda (UNEFM). Santa Ana de Coro. Venezuela

Milton Junior Rivadeneira-Hermida; Juan Carlos Erazo-Álvarez; Katina Vanessa Bermeo-Pazmiño

Diego Patricio Cisneros-Quintanilla

$\underline{\text { DOI } 10.35381 / \mathrm{cm} . v 7 i 12.449}$

\section{Cambios administrativos y organizativos como propuesta emergente en Pandemia}

\section{Administrative and organizational changes as an emerging proposal in Pandemic}

Milton Junior Rivadeneira-Hermida

mjrivadeneirah46@ucacue.edu.ec

Universidad Católica de Cuenca, Cuenca

Ecuador

https://orcid.org/0000-0002-6961-8672

Juan Carlos Erazo-Álvarez

jcerazo@ucacue.edu.ec

Universidad Católica de Cuenca, Cuenca

Ecuador

https://orcid.org/0000-0001-6480-2270

Katina Vanessa Bermeo-Pazmiño kbermeo@ucacue.edu.ec

Universidad Católica de Cuenca, Cuenca

Ecuador

https://orcid.org/0000-0002-4438-7855

Diego Patricio Cisneros-Quintanilla dcisneros@ucacue.edu.ec

Universidad Católica de Cuenca, Cuenca

Ecuador

https://orcid.org/0000-0003-0897-8938

Recibido: 01 de octubre de 2020

Aprobado: 15 de diciembre de 2020 


\title{
RESUMEN
}

El presente artículo tiene como objetivo, plantear un modelo de gestión para la implementación de un cambio de estructura administrativa y organizacional, con miras a las condiciones actuales de teletrabajo, para su realización. Metodológicamente fue de tipo descriptivo. La prefectura de Morona Santiago lleva varios programas y proyectos enmarcados en sus competencias, dentro del desarrollo social del territorio, con este antecedente fue creada la Coordinación, Equidad, Formación y Acción Social que se encarga de la atención a los grupos prioritarios, es prioritario implementar cambios en los temas administrativos y organizativos, con esto se puede garantizar que los beneficiarios mejoren su calidad de vida y que los colaboradores puedan ejercer sus actividades laborales en condiciones de bajo estrés laboral y de seguridad para ellos y sus familias.

Descriptores: Actitud laboral; gestión; dirección de proyecto. (Palabras tomadas del Tesauro UNESCO).

\begin{abstract}
The objective of this article is to propose a management model for the implementation of a change in the administrative and organizational structure, with a view to the current telework conditions, for its realization. Methodologically it was descriptive. The Morona Santiago prefecture has several programs and projects framed within its competencies, within the social development of the territory, with this background the Coordination, Equity, Training and Social Action was created that is responsible for the attention to priority groups, it is a priority to implement changes in administrative and organizational issues, with this it can be guaranteed that the beneficiaries improve their quality of life and that the collaborators can carry out their work activities in conditions of low work stress and safety for themselves and their families.
\end{abstract}

Descriptores: Work attitudes; management; project management. (Words taken from the UNESCO Thesaurus). 


\section{CIENCIAMATRIA}

Revista Interdisciplinaria de Humanidades, Educación, Ciencia y Tecnología

Año VII. Vol. VII. N¹2. Enero - Junio. 2021

Hecho el depósito de ley: pp201602FA4721

ISSN-L: 2542-3029; ISSN: 2610-802X

Universidad Nacional Experimental Francisco de Miranda (UNEFM). Santa Ana de Coro. Venezuela

Milton Junior Rivadeneira-Hermida; Juan Carlos Erazo-Álvarez; Katina Vanessa Bermeo-Pazmiño

Diego Patricio Cisneros-Quintanilla

\section{INTRODUCCIÓN}

La pandemia que vivimos en la actualidad, es el acontecimiento que ha marcado una nueva tendencia de vida y uno de los desafíos más fuertes que hemos enfrentado como humanidad desde que se suscitó la II Guerra Mundial. Desde que comenzó su diseminación en Asia en el 2019, el virus ha invadido el mundo, el número de casos aumentan cada día y hasta la fecha no vemos una posible solución para este aumento de contagios a nivel de la población mundial. EI COVID-19 va más allá de ser una crisis de salud de escala global, tiene el potencial de crear crisis social, económica y política devastadora, en cada país que toca. Cada día que pasa, más y más personas, pierden sus trabajos y fuentes de ingresos, estima que se podrían perder 25 millones de empleos a nivel mundial al finalizar este año (OIT, 2020).

Ante esta situación es imperioso que las empresas públicas y privadas, adopten metodologías y restructuraciones que les permitan afrontar esta crisis sanitaria, sin tener que recurrir al despido masivo de sus colaboradores. En la provincia de Morona Santiago, se encuentran operando varias instituciones del sector público que se dedican a varias áreas de atención a la ciudadanía, una de ellas es la Coordinación, Equidad, Formación y Acción Social CEFAS - Patronato del Gobierno Autónomo Descentralizado de Morona Santiago, que fue creada con el fin de brindar opciones para mejorar la calidad de vida de los grupos vulnerables de la provincia; propender a que se cumplan con los derechos que contempla la constitución en el marco de sus competencias instauradas en el Código Orgánico de Organización Territorial, Autonomía y Descentralización COOTAD en lo referente al ámbito social, estas acciones inciden en el desarrollo humano integral de los beneficiarios, tanto en el ámbito productivo, intercultural, financiera y social, que les permita vivir independencia y paz (Prefectura de Morona Santiago, 2019).

sin embargo, se puso en evidencia que la estructura organizativa y administrativa de dicha entidad, no estuvo preparada para afrontar la nueva normalidad y optimizar las actividades mediante la modalidad de teletrabajo de sus colaboradores. El constante 


\section{CIENCIAMATRIA}

Revista Interdisciplinaria de Humanidades, Educación, Ciencia y Tecnología

Año VII. Vol. VII. N¹2. Enero - Junio. 2021

Hecho el depósito de ley: pp201602FA4721

ISSN-L: 2542-3029; ISSN: 2610-802X

Universidad Nacional Experimental Francisco de Miranda (UNEFM). Santa Ana de Coro. Venezuela

Milton Junior Rivadeneira-Hermida; Juan Carlos Erazo-Álvarez; Katina Vanessa Bermeo-Pazmiño

Diego Patricio Cisneros-Quintanilla

ecosistema cambiante que circunda a las amenazas, obliga a que las mismas estén en capacidad de dar respuesta pronta a cualquier vicisitud que amenace con entorpecer los procesos administrativos y organizacionales, por lo tanto, es vital ofrecer mejores lineamientos estratégicos para gestionar y administrar de forma eficiente los distintos procesos típicos de cada empresa (Pardo-Díaz \& Rodríguez-Martín, 2020).

El presente artículo tiene como objetivo, plantear un modelo de gestión para la implementación de un cambio de estructura administrativa y organizacional, con miras a las condiciones actuales de teletrabajo en el Ecuador.

\section{Referencial Teórico}

\section{Estructura administrativa y organizacional}

Las organizaciones poseen una estructura organizacional que guía sus actividades para lograr sus objetivos, dichas estructuras están organizadas en niveles jerárquicos, funcionales, entre otras. El organigrama funcional de la organización es donde se visualiza la posición que los colaboradores ocupan dentro de la empresa. Este orden permite establecer protocolos de actuación, procesos optimizados, trabajos productivos y resultados controlados.

Una estructura organizacional es donde se expresa las diferentes maneras en las que se pueden dividir las diferentes actividades laborales en diferentes tareas (Mintzberg, 1984). La distribución organizativa de una empresa presenta varias características: se vuelve un proceso permanente y cambiante al transcurrir el tiempo; dependiendo de las decisiones de los altos cargos de la organización puede ser centralizada o descentralizada; se deben especializar las distintas áreas, dependiendo del tamaño de la empresa; coordinación y colaboración entre los departamentos; estandarización de procesos; debe estar alineada con los principios y objetivos de la organización, para esto los objetivos deben ser alcanzables dependiendo de la disponibilidad de recursos y herramientas tecnológicas con las cuales cuente la organización; cada colaborador debe conocer dónde encontrar 
los insumos e información necesaria para ejecutar sus funciones; debe existir un manual de funciones donde se especifique el rol de cada persona dentro de la organización. Los puntos clave para definir la estructura organizacional son la misión y visión, de estas derivan sus modelos de gestión, por ejemplo, las pequeñas empresas por lo general usan una estructura horizontal, esta facilita optar por diferentes decisiones y las modificaciones en sus estrategias, según los requerimientos de sus respectivos mercados, sin embargo, a medida que crecen las empresas, su sistema organizacional se ve dificultado, por este motivo es importante recalcar que una estructura organizacional debe ser continua, adaptable y debe estar en constante evolución. Existen varios modelos de estructuras organizacionales, a continuación, se destacan las principales:

1. Divisional. Las organizaciones se componen de diferentes departamentos y actividades.

2. Funcional. Se basa en los roles de cada colaborador.

3. Matricial. Los equipos tienen diferentes proyectos en los que cada miembro desarrolla una misma función

4. Geográfica. Para empresas que operan en varios países.

5. Central. Existen varios supervisores por cada empleado, generalmente aplicado a empresas de gran tamaño y que cuentan con varias locaciones.

6. Lineal. Es la más antigua, es el modelo donde la cabeza toma todas las decisiones y goza del poder absoluto en la organización.

7. Horizontal. No obedece a un sistema centralizado, confía en todos los miembros del equipo y están en condiciones de tomar decisiones (Pérez, 2015).

Para establecer un sistema organizacional es necesario partir a algunos elementos que sirven de guía para generar un plan que facilite un crecimiento sostenible de la empresa, estos elementos son: cantidad de colaboradores, ubicación geográfica, branding y modelo de negocio. 
Los procesos administrativos - organizacionales y el reto de asumir nuevas modalidades de trabajo se han convertido en un tema de alta incidencia en esta nueva realidad (CEPAL, 2020), con base a la realidad podemos inferir que las cuarentenas forzosas obligaran a las empresas a la virtualización de las relaciones económicas y sociales; en este sentido, el teletrabajo prevalecerá en más países y empresas, provocando que la digitalización avance de manera acelerada.

Las empresas se vieron en la obligación de acatar nuevas estrategias para continuar con su funcionamiento en sus procesos administrativos, comerciales, operativos, con el fin de mantener sus flujos financieros en términos aceptables para garantizar la sostenibilidad de las mismas, para conseguirlo han decidido adoptar la modalidad de teletrabajo y salvaguardar a sus colaboradores, pero se debe considerar que existen sectores donde se imposibilita esta modalidad (por ejemplo en el sector industrial) (Pardo-Díaz \& Rodríguez-Martín, 2020).

\section{Teletrabajo como alternativa laboral en tiempos de pandemia}

Es cuando se presta servicios personales o profesionales de forma lícitos con relación de dependencia, de modalidad a distancia, respetando la jornada laborar establecida con el empleador (Ministerio de Trabajo, 2020). Para la realización del teletrabajo se usa de forma frecuente medios electrónicos para el procesamiento de información, además del uso permanente de medios de telecomunicación para facilitar las interacciones entre el colaborador y la organización.

Esta modalidad presenta varios beneficios, tanto para la empresa, como para el colaborador, más aún en las condiciones actuales de distanciamiento social, se presenta la reducción de los desplazamientos en espacios públicos; se presenta una mayor flexibilidad del horario laboral; facilita la autonomía (CEPAL, 2020).

Existen varios tipos de teletrabajo: trabajo en domicilio, hoy en día es el tipo con mayor potencial de crecimiento, esto debido a la crisis sanitaria, implica el uso de medios 
electrónicos, se usa de forma general cuando los colaboradores se encargan del procesamiento de datos; los centros de teletrabajo, son oficinas donde se comparten los insumos y equipos entre los colaboradores, se estima que esta modalidad es más amigable que la anterior.

Los centros de teletrabajo poseen las siguientes particularidades: disminuye el tiempo de movilización y recursos para los colaboradores; facilitan que los residentes de áreas rurales accedan a diferentes tipos de trabajos; proporciona la solución para los colaboradores que se encuentran en los grupos vulnerables en temas de salud; presentan un mejor aprovechamiento de los lugares de trabajo, dado que los mismos pueden ser utilizados durante todo el día en turnos diferentes (Ministerio de Salud de Costa Rica, 2020).

El emplazamiento de este tipo de centros se justifica para las empresas privadas, cuando sus trabajadores invierten más de una hora diaria en su desplazamiento, en el caso de las instituciones públicas, la financiación de este tipo de centros se explica fundamentalmente por temas de formación, educación y creación de empleo. Teletrabajado móvil o itinerante, utiliza redes telefónicas y radiofónicas, en la actualidad, presenta un crecimiento considerable, gracias a los adelantos tecnológicos.

Ante la nueva normalidad, es poco probable que el teletrabajo desaparezca, esto sumado a los problemas de tráfico en aumento, crecimiento de las ciudades, revolución informática y demás exigencias de las sociedades crecientes, las empresas se ven obligadas a reorganizarse y contratar a personal más cualificado, mejorar el servicio al cliente y reducir los costos fijos (Ministerio de Salud de Costa Rica, 2020).

En el Ecuador, se puede definir al teletrabajo según lo establecido en los Acuerdos Ministeriales No. MDT-2016-190, para el sector privado y para el sector público en el MDT-2017-090. Sector privado, se considera como una forma de prestar servicios de manera no presencial en jornadas de trabajo ordinarias y especiales por medio de las cuales el trabajador realiza tareas determinadas fuera de las instalaciones del empleador, 


\section{CIENCIAMATRIA}

Revista Interdisciplinaria de Humanidades, Educación, Ciencia y Tecnología

Año VII. Vol. VII. N¹2. Enero - Junio. 2021

Hecho el depósito de ley: pp201602FA4721

ISSN-L: 2542-3029; ISSN: 2610-802X

Universidad Nacional Experimental Francisco de Miranda (UNEFM). Santa Ana de Coro. Venezuela

Milton Junior Rivadeneira-Hermida; Juan Carlos Erazo-Álvarez; Katina Vanessa Bermeo-Pazmiño

Diego Patricio Cisneros-Quintanilla

siempre y cuando las necesidades y la naturaleza del trabajo lo ameriten, utilizando las tecnologías de información y comunicación conocidas como TIC, tanto como para su administración como para su gestión y control.

Por lo tanto, el teletrabajo puede prestarse de las siguientes formas:

1. Permanente: Son actividades realizadas siempre fuera de las instalaciones, utilizando todos los recursos tecnológicos y de información disponibles, el teletrabajador deberá asistir a las instalaciones de la organización siempre y cuando su presencia sea requerida por el empleador.

2. Parcial: Se realizan fuera del lugar habitual de trabajo hasta un máximo de 24 horas a la semana, por lo que el resto de horas deberán ser dentro de las instalaciones.

Teletrabajador se define como una persona que tiene calidad de trabajador de conformidad con lo dispuesto en el Código de Trabajo, y que efectúe sus tareas mediante el teletrabajo, que se desarrolla fuera de las instalaciones en las que normalmente realizaría sus labores. El sitio habitual del empleador es el lugar físico donde la parte emprendedora desarrolla sus actividades, ya sea la matriz o cualquiera de sus sucursales.

El contrato de teletrabajo contiene una descripción amplia de las labores que deberá realizar, condiciones de ejecución, salario y demás beneficios de ley; identificación de herramientas de trabajo que utilizará el teletrabajador y determinación de la parte responsable de la provisión, instalación y mantenimiento de equipos; la unidad departamental a la cual pertenece el teletrabajador de ser así el caso, como el señalamiento del nombre y cargo de su inmediato superior; en el caso de los teletrabajadores parciales, los días en los cuales se efectuará el trabajo, modalidades de entrega de evidencias y demás particularidades de trabajo dependiendo del cargo y de las labores que desempeñe. 
La naturaleza del teletrabajo asegura que la forma de prestación de servicios es de manera libre y voluntaria y para su implementación debe existir un acuerdo entre las partes involucradas, el cual deberá constar por escrito en el contrato que suscriba. El teletrabajo se puede entender como parte de la descripción inicial de la modalidad de trabajo o puede integrarse posteriormente. (Ministerio de Trabajo, 2016).

Sector público, se entiende como la prestación de servicios de carácter no presencial en jornadas ordinarias y especiales de trabajo, por medio de la cual el/la servidora pública realiza sus tareas fuera del área de trabajo para la cual presta sus servicios, siempre que los requerimientos y la naturaleza del servicio lo requieran. Se entiende como teletrabajador a aquellas personas que tienen calidad de servidor público de conformidad con lo dispuesto en la Ley Orgánica de Servicio Público LOSEP, con excepción de los puestos de alto nivel, y que efectúe sus tareas mediante el teletrabajo fuera del espacio físico de la organización (Presidencia de la República del Ecuador, 2010).

Los colaboradores que realicen actividades laborales mediante la modalidad de teletrabajo, tendrán los mismos derechos y obligaciones que los que lo hagan de forma presencial, en los dos casos, son los responsables del uso de la información, entregada o generada durante la ejecución de sus labores. La jornada laboral no podrá sobrepasar los límites establecidos en el Código de Trabajo, sin embargo, el horario de trabajo podrá ser modificado según las conveniencias de las partes. Los contratos o adendas a contratos de trabajo elaborados para contratar personal mediante esta modalidad, deben ser por escrito, siendo respetada, por la parte empleadora, lo dispuesto en el numeral 7 del artículo 42 del Código de Trabajo (Ministerio de Trabajo, 2017).

\section{Acciones ante la situación actual de la pandemia provocada por el COVID 19.}

En los actuales momentos de incertidumbre y preocupación, por la realidad que se vive en el mundo, no se trata sólo de preguntar qué hacen los líderes de las empresas, sino también cómo lo hacen. El COVID-19 ha tomado al mundo por sorpresa, causando 
incertidumbre y desacomodo en el desarrollo de las actividades que se habían convertido en cotidianas para la humanidad.

Las personas son el patrimonio más importante de las empresas, por lo tanto, se debe planear cómo responder a las necesidades de la gente durante la actual crisis sanitaria, para lograrlo es importante pensar en las tres dimensiones profundamente conectadas en una organización: Trabajo (el qué), Fuerza laboral (el quién), y Lugar de trabajo (el dónde).

Para el planteamiento de propuestas emergentes que apuntalen la gestión de las empresas, en primera instancia, es priorizar a las personas mientras dure este estado de emergencia, se debe conformar equipos para evaluar las estrategias que abordan el impacto y los riesgos a los cuales se ve sometida la fuerza laboral de las empresas para el desarrollo de acciones apropiadas, según cada escenario que se presente.A mediano plazo, esta situación sanitaria puede presentarse como una oportunidad para crear fuerzas laborales resilientes, enfocadas en la salud y bienestar de los colaboradores de las empresas. A continuación, se citan algunas estrategias para responder el desafío de las empresas al adaptarse a la nueva realidad, poniendo a las personas por sobre los capitales:

1. Establecer una oficina de respuesta y continuidad del negocio.

2. Confirmar roles críticos y planes de contingencia.

3. Evaluar el trabajo real de su empresa y cómo podría cambiarse.

4. Comprender qué trabajo es de misión crítica y qué se puede aplazar o cambiar de prioridad.

5. Desarrollar planes para la fuerza laboral directa e indirecta.

6. Definir la estrategia de comunicación y hacerla visible.

7. Educar a los empleados sobre los síntomas y la prevención del COVID-19.

8. Establecer procedimientos de apoyo a los empleados.

9. Desarrollar estrategias para reducción temporal de la fuerza laboral. 
CIENCIAMATRIA

Revista Interdisciplinaria de Humanidades, Educación, Ciencia y Tecnología

Año VII. Vol. VII. N¹2. Enero - Junio. 2021

Hecho el depósito de ley: pp201602FA4721

ISSN-L: 2542-3029; ISSN: 2610-802X

Universidad Nacional Experimental Francisco de Miranda (UNEFM). Santa Ana de Coro. Venezuela

Milton Junior Rivadeneira-Hermida; Juan Carlos Erazo-Álvarez; Katina Vanessa Bermeo-Pazmiño

Diego Patricio Cisneros-Quintanilla

10. Preparar planes para la interrupción del lugar de trabajo y su reactivación.

11. Desarrollar protocolos y obligaciones claros para los empleados que están en riesgo.

12. Revise las políticas de licencia por enfermedad.

13. Prepararse para un mayor ausentismo y fuerza laboral renuente.

14. Revisar las políticas y prácticas contra la discriminación.

15. Preparar el lugar de trabajo para la contención y la contaminación.

16. Actualizar los protocolos de viaje y reunión.

17. Considerar las fuentes de "noticias" e información en el lugar de trabajo (Picatoste, 2020).

\section{MÉTODO}

Para ejecutar el presente trabajo de investigación se ha utilizado la metodología de tipo descriptiva con diseño no experimental transversal, dado a que el estudio de las variables dependientes e independientes se realizó sin usanza arbitraria en su medio natural, para luego desarrollar su análisis y determinación de resultados, recopilándose la información en único momento. Abordándose una muestra poblacional de 31 funcionarios de Coordinación, Equidad, Formación y Acción Social, a través del muestreo por conveniencia, aplicándose una encuesta online y cuestionario de diversas alternativas de respuestas, siendo validado por juicio de expertos, calculándose la fiabilidad de Alfa de Cronbach en 0,86 validándose como confiable para su aplicación. La información recopilada fue procesada a través de la estadística descriptiva. 
CIENCIAMATRIA

Revista Interdisciplinaria de Humanidades, Educación, Ciencia y Tecnología

Año VII. Vol. VII. N¹2. Enero - Junio. 2021

Hecho el depósito de ley: pp201602FA4721

ISSN-L: 2542-3029; ISSN: 2610-802X

Universidad Nacional Experimental Francisco de Miranda (UNEFM). Santa Ana de Coro. Venezuela

Milton Junior Rivadeneira-Hermida; Juan Carlos Erazo-Álvarez; Katina Vanessa Bermeo-Pazmiño

Diego Patricio Cisneros-Quintanilla

\section{RESULTADOS}

El 93,5\% conoce cuales son los objetivos que persigue la organización, la califican como buena $(90,3 \%)$. En el ámbito de la crisis sanitaria que enfrentamos, el 80,6\% considera que la estructura organizacional fue buena, además el $67,7 \%$ siente que está seguro en el ámbito laboral a pesar de la pandemia, existe el $96,8 \%$ de conocimiento de lo que es el teletrabajo.

El $61,3 \%$ de los colaboradores encuestados consideran que el teletrabajo les representa beneficios en la actualidad y que no afecta sus condiciones psicológicas ni emocionales $(67,7 \%)$, sin embargo, ante lo expuesto el $83,9 \%$ considera que es importante reestructurar el modelo organizativo ante la nueva normalidad.

Se solicitó a los encuestados que colaboren con ideas para mejorar la estructura organizacional, entre lo recopilado se puede citar: capacitaciones permanentes, comunicación entre los distintos departamentos, planificación de proyectos, reorganización de actividades, priorizar adquisiciones, seguimiento a las adquisiciones, valoración del desempeño laboral, trabajo en campo, atención a personas vulnerables, mejorar el flujo de los procesos, fortalecer las áreas que son eje de promoción del trabajo humanitario.

Solicitar el apoyo técnico de los profesionales de campo para incluir proyectos de trabajo, crear nuevos programas de ayuda social y emprendedores, evitar el papeleo entre departamentos, organizar de mejor manera las actividades de teletrabajo para que el funcionario no haga doble trabajo ni horarios extenuantes; es decir que todo sea online sin acudir a las oficinas, brindar capacitación sobre el uso de herramientas digitales para adaptar el trabajo a la nueva normalidad y de forma segura, y, fortalecer la relación entre compañeros, crear empatía, con incentivos (una felicitación pública, un reconocimiento) para motivar a los funcionarios y todos trabajen por el bien común y en equipo. 


\section{CIENCIAMATRIA}

Revista Interdisciplinaria de Humanidades, Educación, Ciencia y Tecnología

Año VII. Vol. VII. N¹2. Enero - Junio. 2021

Hecho el depósito de ley: pp201602FA4721

ISSN-L: 2542-3029; ISSN: 2610-802X

Universidad Nacional Experimental Francisco de Miranda (UNEFM). Santa Ana de Coro. Venezuela

Milton Junior Rivadeneira-Hermida; Juan Carlos Erazo-Álvarez; Katina Vanessa Bermeo-Pazmiño

Diego Patricio Cisneros-Quintanilla

\section{PROPUESTA}

La propuesta de cambios administrativos y organizativos como propuesta emergente en tiempos COVID, servirá como insumo para mejorar la capacidad de respuesta de la organización ante posibles amenazas y garantizar que los objetivos planteados sean cumplidos y el personal tenga las facilidades para realizar sus actividades.

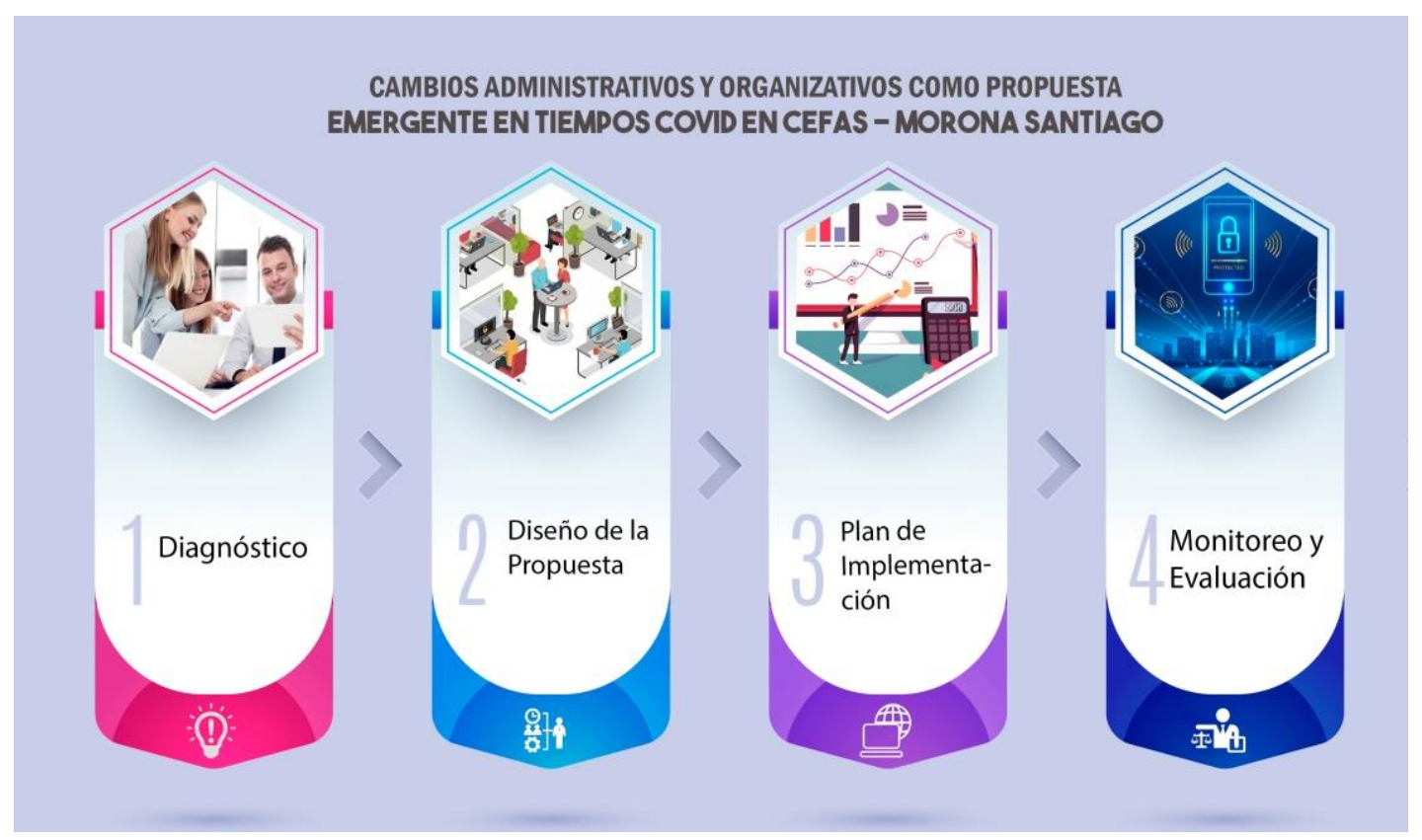

Figura 1. Esquema de la propuesta cambios administrativos y organizativos como propuesta emergente en tiempos COVID en CEFAS - Morona Santiago

\section{Diagnóstico}

Para realizar la propuesta planteada en el trabajo en mención, se debe elaborar un diagnóstico del estado inicial, con el fin de tener los insumos necesarios para el planteamiento de las acciones requeridas para lograr los objetivos. 
CIENCIAMATRIA

Revista Interdisciplinaria de Humanidades, Educación, Ciencia y Tecnología

Año VII. Vol. VII. N¹2. Enero - Junio. 2021

Hecho el depósito de ley: pp201602FA4721

ISSN-L: 2542-3029; ISSN: 2610-802X

Universidad Nacional Experimental Francisco de Miranda (UNEFM). Santa Ana de Coro. Venezuela

Milton Junior Rivadeneira-Hermida; Juan Carlos Erazo-Álvarez; Katina Vanessa Bermeo-Pazmiño Diego Patricio Cisneros-Quintanilla

En la tabla 1 se analiza el estado de situación inicial de la organización mediante un análisis FODA, tomando como referencia los factores que inciden de manera interna y externa en la misma.

Tabla 1.

Análisis organizacional FODA.

\begin{tabular}{|l|c|c|c|}
\hline $\begin{array}{l}\text { Factores internos determinantes de } \\
\text { éxito }\end{array}$ & Peso & Calificación & Peso ponderado \\
\hline Fortalezas & & & \\
\hline Infraestructura de calidad & 0,15 & 4 & 0,60 \\
\hline Experiencia en temas de ayuda social & 0,20 & 4 & 0,80 \\
\hline Legislación favorable & 0,05 & 3 & 0,15 \\
\hline Personal capacitado & 0,10 & 4 & 0,40 \\
\hline Debilidades & 0,15 & 1 & 0,15 \\
\hline Recorte de personal & 0,05 & 2 & 0,10 \\
\hline Bajo impacto en actividades sociales & 0,10 & 1 & 0,10 \\
\hline $\begin{array}{l}\text { Limitados recursos para capacitadores } \\
\text { expertos }\end{array}$ & 0,10 & 1 & 0,10 \\
\hline $\begin{array}{l}\text { Baja disponibilidad de equipos para } \\
\text { capacitaciones }\end{array}$ & 0,10 & 2 & 0,20 \\
\hline $\begin{array}{l}\text { Falta de mapeo de beneficiarios en la } \\
\text { provincia }\end{array}$ & $\mathbf{1 , 0 0}$ & & $\mathbf{2 , 6 0}$ \\
\hline Total
\end{tabular}

\begin{tabular}{|l|c|c|c|}
\hline $\begin{array}{l}\text { Factores externos determinantes de } \\
\text { éxito }\end{array}$ & Peso & Calificación & $\begin{array}{c}\text { Peso } \\
\text { ponderado }\end{array}$ \\
\hline Oportunidades & & & \\
\hline $\begin{array}{l}\text { Financiamiento para nuevos proyectos y } \\
\text { programas }\end{array}$ & 0,20 & 3 & 0,60 \\
\hline Vinculación con la academia & 0,10 & 3 & 0,30 \\
\hline Legislación favorable & 0,05 & 2 & 0,10 \\
\hline $\begin{array}{l}\text { Programas sociales del gobierno central } \\
\text { sin financiamiento }\end{array}$ & 0,10 & 1 & 0,10 \\
\hline Amenazas & & & \\
\hline Rebrotes de COVID & 0,20 & 1 & 0,20 \\
\hline
\end{tabular}




\section{CIENCIAMATRIA}

Revista Interdisciplinaria de Humanidades, Educación, Ciencia y Tecnología

Año VII. Vol. VII. No12. Enero - Junio. 2021

Hecho el depósito de ley: pp201602FA4721

ISSN-L: 2542-3029; ISSN: 2610-802X

Universidad Nacional Experimental Francisco de Miranda (UNEFM). Santa Ana de Coro. Venezuela

Milton Junior Rivadeneira-Hermida; Juan Carlos Erazo-Álvarez; Katina Vanessa Bermeo-Pazmiño Diego Patricio Cisneros-Quintanilla

\begin{tabular}{|l|l|l|l|}
$\begin{array}{l}\text { Falta de aceptación por parte de los } \\
\text { beneficiarios }\end{array}$ & 0,15 & 2 & 0,30 \\
\hline Vías de segundo orden en mal estado & 0,05 & 4 & 0,20 \\
\hline Falta de recursos económicos & 0,15 & 2 & 0,30 \\
\hline Total & $\mathbf{1 , 0 0}$ & & $\mathbf{2 , 1 0}$ \\
\hline
\end{tabular}

Fuente: Elaboración propia.

\section{Gráfico de situación FODA}

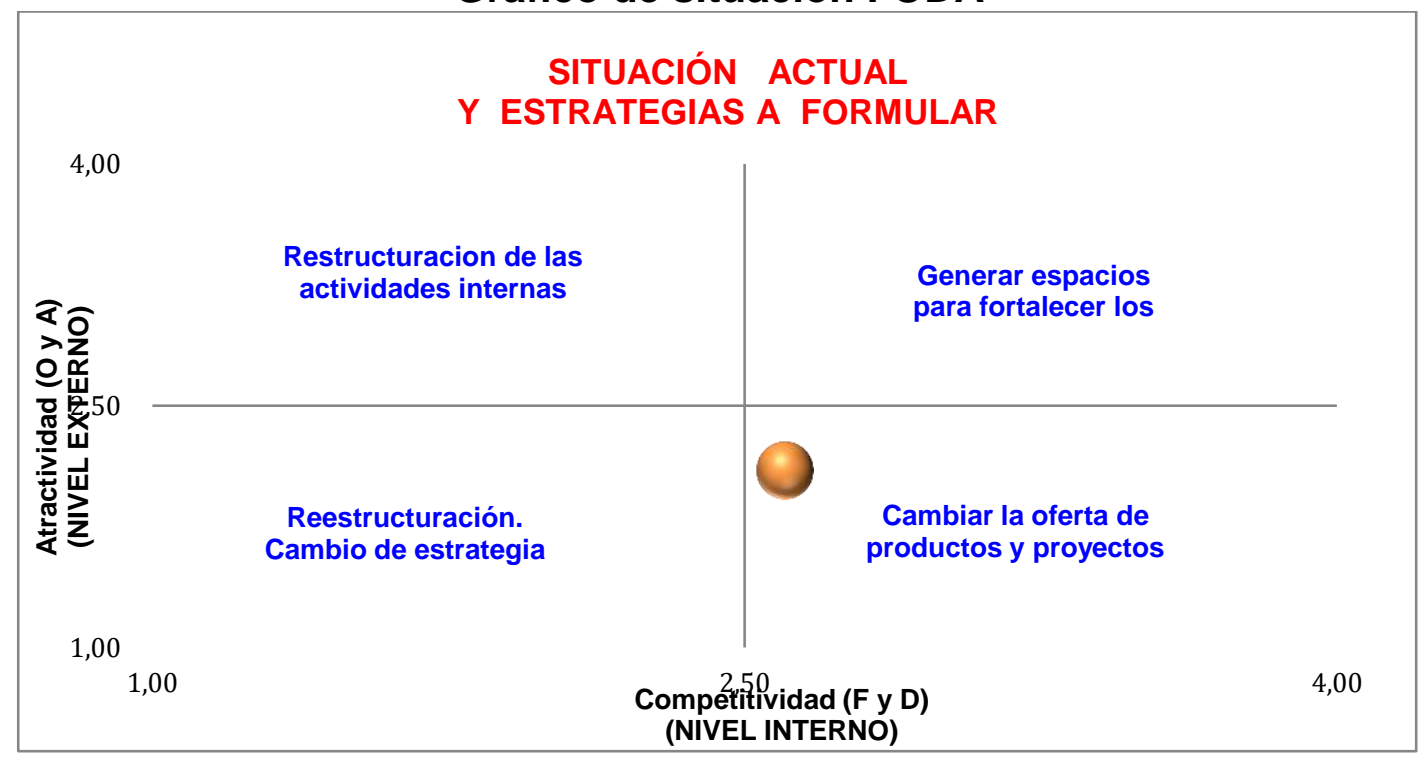

Figura 2. Grafico de situación actual FODA.

Fuente: Elaboración propia. 


\section{CIENCIAMATRIA}

Revista Interdisciplinaria de Humanidades, Educación, Ciencia y Tecnología

Año VII. Vol. VII. N¹2. Enero - Junio. 2021

Hecho el depósito de ley: pp201602FA4721

ISSN-L: 2542-3029; ISSN: 2610-802X

Universidad Nacional Experimental Francisco de Miranda (UNEFM). Santa Ana de Coro. Venezuela

Milton Junior Rivadeneira-Hermida; Juan Carlos Erazo-Álvarez; Katina Vanessa Bermeo-Pazmiño

Diego Patricio Cisneros-Quintanilla

\section{Diseño de la propuesta}

Los sistemas organizacionales de la actualidad son muy competitivos, por lo tanto, se debe buscar un modelo que se adapte a la realidad de cada organización y aporte con la consecución de los objetivos planteados, la propuesta de cambios administrativos y organizativos qué se detalla a continuación:

\section{Flujograma de cambios administrativos y organizativos}

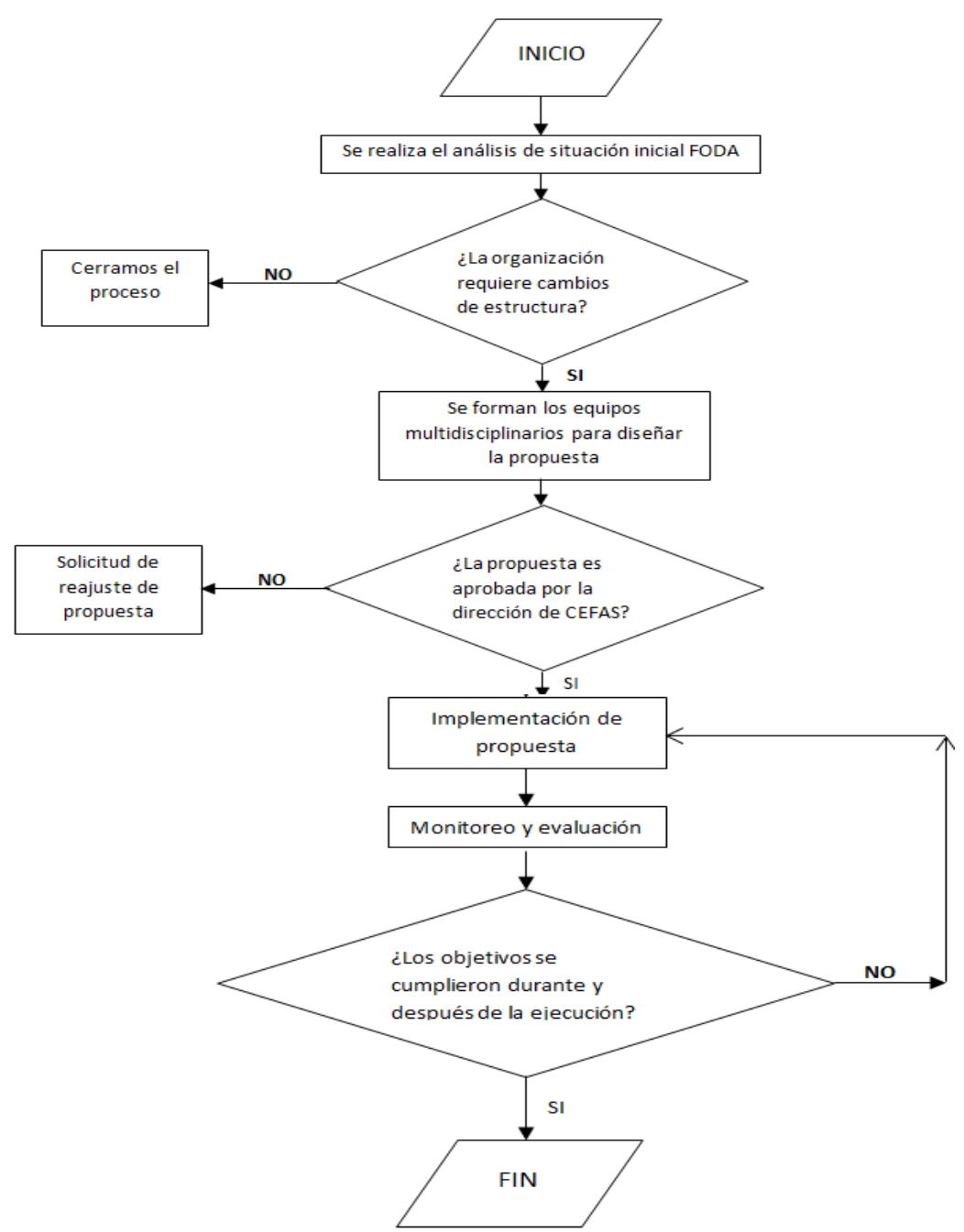

Figura 3. Flujograma de cambios administrativos.

Fuente: Elaboración propia. 
CIENCIAMATRIA

Revista Interdisciplinaria de Humanidades, Educación, Ciencia y Tecnología

Año VII. Vol. VII. N¹2. Enero - Junio. 2021

Hecho el depósito de ley: pp201602FA4721

ISSN-L: 2542-3029; ISSN: 2610-802X

Universidad Nacional Experimental Francisco de Miranda (UNEFM). Santa Ana de Coro. Venezuela

Milton Junior Rivadeneira-Hermida; Juan Carlos Erazo-Álvarez; Katina Vanessa Bermeo-Pazmiño

Diego Patricio Cisneros-Quintanilla

\section{Plan de implementación}

Dentro de la propuesta para cambios administrativos es importante identificar el plan de implementación que será necesario para garantizar el adecuado funcionamiento y operación de la organización con su adecuado soporte administrativo y organizativo. La matriz de análisis para la implementación propuesta a continuación nos da una perspectiva clara sobre el avance en los componentes planteados para la ejecución del presente análisis.

Es importante determinar el grado de avance de cada uno de los componentes de las diferentes etapas, esto queda a consideración del evaluador, quien dará un grado porcentual de cumplimiento a cada una.

Tabla 2.

Plan de implementación.

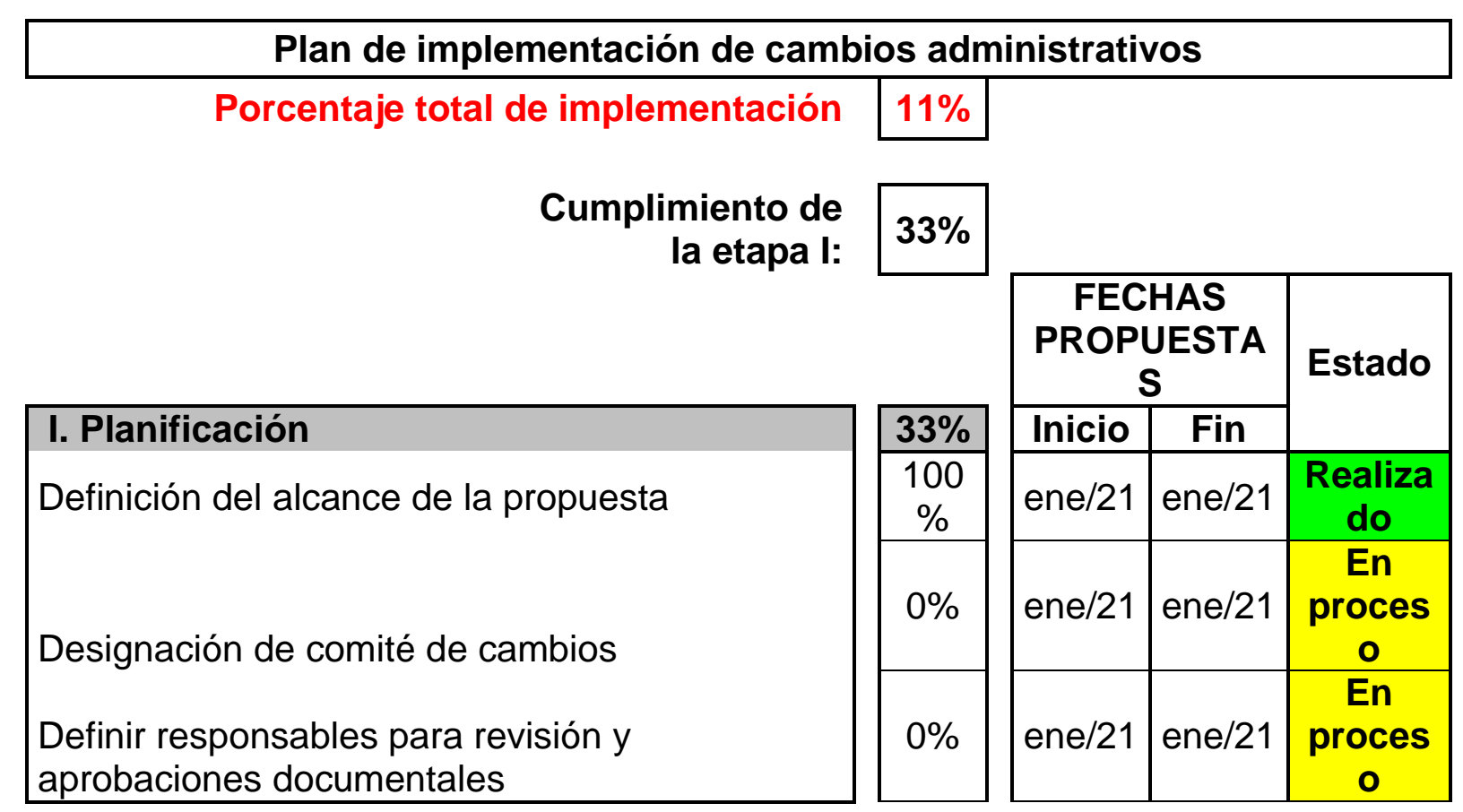


Universidad Nacional Experimental Francisco de Miranda (UNEFM). Santa Ana de Coro. Venezuela

Milton Junior Rivadeneira-Hermida; Juan Carlos Erazo-Álvarez; Katina Vanessa Bermeo-Pazmiño Diego Patricio Cisneros-Quintanilla

\section{Total, de cumplimiento de la etapa II:}

\section{Implementación}

\section{Gestión de calidad en cambios} administrativos

Elaboración del manual de procesos

Identificación de los procesos externos en el manual

Aprobación del manual

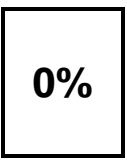

\section{$0 \%$}

\begin{tabular}{|c|c|c|c|}
\hline & $\begin{array}{r}\text { FEC } \\
\text { PROPI }\end{array}$ & $\begin{array}{l}\text { HAS } \\
\text { JESTA } \\
\text { jESTA }\end{array}$ & Estado \\
\hline $0 \%$ & Inicio & Fin & \\
\hline $0 \%$ & ene/21 & feb/21 & $\begin{array}{c}\text { En } \\
\text { proces } \\
0\end{array}$ \\
\hline $0 \%$ & feb/21 & $\mathrm{mar} / 21$ & $\begin{array}{c}\text { En } \\
\text { proces } \\
0\end{array}$ \\
\hline $0 \%$ & $\mathrm{mar} / 21$ & $\mathrm{mar} / 21$ & $\begin{array}{c}\text { En } \\
\text { proces } \\
0\end{array}$ \\
\hline
\end{tabular}

\begin{tabular}{|c|c|c|c|}
\hline & $\begin{array}{r}\text { FEC } \\
\text { PROPI }\end{array}$ & $\begin{array}{l}\text { HAS } \\
\text { JESTA } \\
\text { jESTA }\end{array}$ & Estado \\
\hline $0 \%$ & Inicio & Fin & \\
\hline $0 \%$ & ene/21 & feb/21 & $\begin{array}{c}\text { En } \\
\text { proces } \\
0\end{array}$ \\
\hline $0 \%$ & feb/21 & $\mathrm{mar} / 21$ & $\begin{array}{c}\text { En } \\
\text { proces } \\
0\end{array}$ \\
\hline $0 \%$ & $\mathrm{mar} / 21$ & $\mathrm{mar} / 21$ & $\begin{array}{c}\text { En } \\
\text { proces } \\
0\end{array}$ \\
\hline
\end{tabular}

\section{Responsabilidad de la dirección \\ Enfoque de los beneficiarios}

Definición de las estrategias a implementar en CEFAS

Difusión de las estrategias

Definición de objetivos estratégicos 
CIENCIAMATRIA

Revista Interdisciplinaria de Humanidades, Educación, Ciencia y Tecnología

Año VII. Vol. VII. N¹2. Enero - Junio. 2021

Hecho el depósito de ley: pp201602FA4721

ISSN-L: 2542-3029; ISSN: 2610-802X

Universidad Nacional Experimental Francisco de Miranda (UNEFM). Santa Ana de Coro. Venezuela

Milton Junior Rivadeneira-Hermida; Juan Carlos Erazo-Álvarez; Katina Vanessa Bermeo-Pazmiño Diego Patricio Cisneros-Quintanilla

Definición de roles y funciones del personal

Determinación de la frecuencia de la revisión por la dirección

Elaboración de la revisión por la dirección

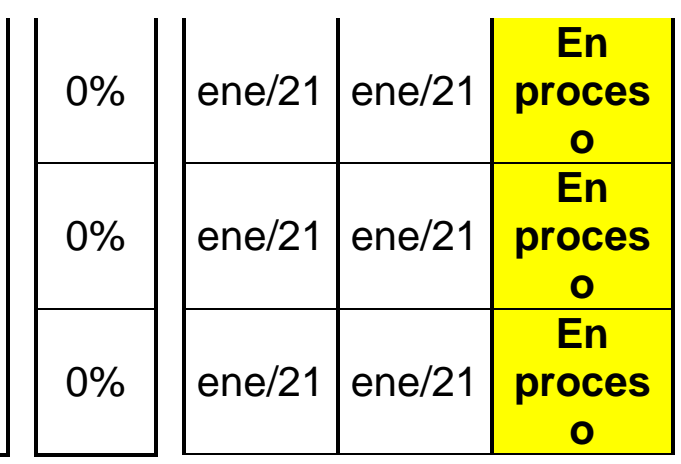

\section{Gestión de los recursos}

Provisión de los recursos por parte de Prefectura

Definir planes de mantenimiento preventivo para infraestructura, equipos y vehículos.

Registros de educación, formación, experiencia y habilidades de los colaboradores

Definir plan de capacitación

Definir evaluación de eficacia de las capacitaciones

\section{Cumplimiento de la etapa III:}

\begin{tabular}{|c|c|c|c|}
\hline & $\begin{array}{r}\text { FEC } \\
\text { PROPL }\end{array}$ & $\begin{array}{l}\text { HAS } \\
\text { JESTA } \\
\text {; }\end{array}$ & Estado \\
\hline $0 \%$ & Inicio & Fin & \\
\hline $0 \%$ & ene/21 & ene/21 & $\begin{array}{c}\text { En } \\
\text { proces } \\
0\end{array}$ \\
\hline $0 \%$ & ene/21 & ene/21 & $\begin{array}{c}\text { En } \\
\text { proces } \\
0\end{array}$ \\
\hline $0 \%$ & ene/21 & ene/21 & $\begin{array}{c}\text { En } \\
\text { proces } \\
0\end{array}$ \\
\hline $0 \%$ & ene/21 & ene/21 & $\begin{array}{c}\text { En } \\
\text { proces } \\
0\end{array}$ \\
\hline $0 \%$ & ene/21 & ene/21 & $\begin{array}{c}\text { En } \\
\text { proces } \\
0\end{array}$ \\
\hline
\end{tabular}
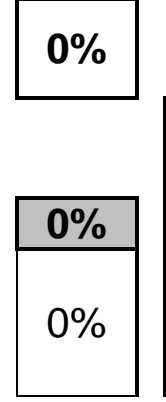

\begin{tabular}{|c|c|c|}
\hline \multicolumn{2}{|c|}{$\begin{array}{c}\text { Fechas } \\
\text { Propuestas }\end{array}$} & \multirow{2}{*}{ Estado } \\
\cline { 1 - 2 } Inicio & Fin & \\
\hline ene/21 & feb/21 & $\begin{array}{c}\text { En } \\
\text { proces } \\
\text { o }\end{array}$ \\
\hline
\end{tabular}


Universidad Nacional Experimental Francisco de Miranda (UNEFM). Santa Ana de Coro. Venezuela

Milton Junior Rivadeneira-Hermida; Juan Carlos Erazo-Álvarez; Katina Vanessa Bermeo-Pazmiño Diego Patricio Cisneros-Quintanilla

\begin{tabular}{|c|c|c|c|c|}
\hline Revisión y aprobación de acciones correctivas & $0 \%$ & ene/21 & feb/21 & $\begin{array}{l}\text { En } \\
\text { proces } \\
0\end{array}$ \\
\hline Revisión y aprobación de acciones preventivas & $0 \%$ & ene/21 & feb/21 & $\begin{array}{c}\text { En } \\
\text { proces } \\
0\end{array}$ \\
\hline Implementación de programa de comunicación & $0 \%$ & $\operatorname{mar} / 21$ & $\begin{array}{c}\text { may/2 } \\
1\end{array}$ & $\begin{array}{c}\text { En } \\
\text { proces } \\
0\end{array}$ \\
\hline Implementación de planes de mejora continua & $0 \%$ & $\begin{array}{c}\mathrm{may} / 2 \\
1\end{array}$ & ago/21 & $\begin{array}{c}\text { En } \\
\text { proces } \\
0\end{array}$ \\
\hline $\begin{array}{l}\text { Capacitación al personal cambios } \\
\text { administrativos }\end{array}$ & $0 \%$ & $\operatorname{sep} / 21$ & $\operatorname{sep} / 21$ & $\begin{array}{c}\text { En } \\
\text { proces } \\
0\end{array}$ \\
\hline $\begin{array}{l}\text { Revisión y aprobación de procedimiento de } \\
\text { control interno }\end{array}$ & $0 \%$ & oct/21 & oct/21 & $\begin{array}{c}\text { En } \\
\text { proces } \\
0\end{array}$ \\
\hline $\begin{array}{l}\text { Elaboración de programa anual de control } \\
\text { interno }\end{array}$ & $0 \%$ & oct/21 & oct/21 & $\begin{array}{c}\text { En } \\
\text { proces } \\
0\end{array}$ \\
\hline Elaboración de informe de no conformidades & $0 \%$ & oct/21 & oct/21 & $\begin{array}{c}\text { En } \\
\text { proces } \\
0\end{array}$ \\
\hline Cierre de no conformidades & $0 \%$ & oct/21 & oct/21 & $\begin{array}{c}\text { En } \\
\text { proces } \\
0\end{array}$ \\
\hline
\end{tabular}

Fuente: Elaboración propia.

\section{Monitoreo y evaluación}

A continuación, se presenta una matriz que muestra el comportamiento de las diferentes etapas de implementación de cambios administrativos en la que se detalla la duración de cada una, además su dependencia para con las demás.

Cada tarea predecesora está enlazada a otra por un tipo específico de dependencia y un tiempo de retardo, cada tarea predecesora está representada por un número, que puede estar seguido por un tipo de dependencia y por el tiempo de adelanto o de retraso. Los 
tipos de dependencia de las predecesoras son FC (fin a comienzo), FF (fin a fin), CC (comienzo a comienzo) y CF (comienzo a fin) (Microsoft Project, 2020). En la columna de días de dependencia, cuando se especifica el tiempo de adelanto, se escribe un número negativo y en el caso de posposición, se escribe un número positivo (Project Management Institute, 2013).

\section{Tabla 3.}

Monitoreo y control.

\section{Matriz para monitoreo y control}

\section{Proyecto: Cambios administrativos y}

Fecha de 01/01

inicio: /2021

Días

planeados de 250

trabajo:

Fecha de fin: $\begin{aligned} & 08 / 09 \\ & / 2021\end{aligned}$

\begin{tabular}{|c|c|c|c|c|c|c|c|c|c|c|}
\hline$\underset{0}{\mathbf{N}}$ & $\begin{array}{l}\text { Descripción } \\
\text { de la etapa }\end{array}$ & $\begin{array}{l}\text { Dura } \\
\text { ción } \\
\text { de la } \\
\text { etapa } \\
\text { (días) }\end{array}$ & $\begin{array}{l}\text { Tarea } \\
\text { depen } \\
\text { diente }\end{array}$ & $\begin{array}{c}\text { Tipo } \\
\text { de } \\
\text { Depen } \\
\text { dencia }\end{array}$ & $\begin{array}{c}\text { Días } \\
\text { de } \\
\text { depen } \\
\text { dencia }\end{array}$ & $\begin{array}{l}\text { Comi } \\
\text { enzo }\end{array}$ & Fin & $\begin{array}{c}\text { Respons } \\
\text { able }\end{array}$ & Estatus & $\begin{array}{c}\text { Fecha } \\
\text { de } \\
\text { finaliz } \\
\text { ación }\end{array}$ \\
\hline 1 & $\begin{array}{l}\text { Diagnóstico } \\
\text { inicial }\end{array}$ & 15 & $\begin{array}{l}\text { No } \\
\text { Aplica }\end{array}$ & $\begin{array}{l}\text { No } \\
\text { Aplica }\end{array}$ & +0 & $\begin{array}{l}01 / 01 \\
/ 21\end{array}$ & $\begin{array}{l}15 / 0 \\
1 / 21\end{array}$ & Dirección & $\begin{array}{c}\text { Completa } \\
\text { do }\end{array}$ & $\begin{array}{c}03 / 11 / \\
17\end{array}$ \\
\hline 2 & $\begin{array}{l}\text { Diseño de la } \\
\text { propuesta }\end{array}$ & 15 & 1 & $\mathrm{CC}$ & +15 & $\begin{array}{c}16 / 01 \\
/ 21\end{array}$ & $\begin{array}{l}30 / 0 \\
1 / 21\end{array}$ & $\begin{array}{l}\text { Equipo } \\
\text { de } \\
\text { planeació } \\
\text { n }\end{array}$ & $\begin{array}{c}\text { No } \\
\text { comenza } \\
\text { do }\end{array}$ & \\
\hline 3 & $\begin{array}{l}\text { Plan de } \\
\text { implementac } \\
\text { ión }\end{array}$ & 15 & 2 & $\mathrm{CC}$ & +15 & $\begin{array}{c}31 / 01 \\
/ 21\end{array}$ & $\begin{array}{l}14 / 0 \\
2 / 21\end{array}$ & $\begin{array}{l}\text { Equipo } \\
\text { de } \\
\text { planeació } \\
\text { n }\end{array}$ & $\begin{array}{c}\text { No } \\
\text { comenza } \\
\text { do }\end{array}$ & \\
\hline
\end{tabular}




\section{CIENCIAMATRIA}

Revista Interdisciplinaria de Humanidades, Educación, Ciencia y Tecnología

Año VII. Vol. VII. N¹2. Enero - Junio. 2021

Hecho el depósito de ley: pp201602FA4721

ISSN-L: 2542-3029; ISSN: 2610-802X

Universidad Nacional Experimental Francisco de Miranda (UNEFM). Santa Ana de Coro. Venezuela

Milton Junior Rivadeneira-Hermida; Juan Carlos Erazo-Álvarez; Katina Vanessa Bermeo-Pazmiño

Diego Patricio Cisneros-Quintanilla

\begin{tabular}{|c|c|c|c|c|c|c|c|c|c|}
\hline 4 & $\begin{array}{l}\text { Monitoreo y } \\
\text { evaluación }\end{array}$ & 200 & 3 & CC & -15 & $\begin{array}{c}16 / 01 \\
/ 21\end{array}$ & $\begin{array}{l}03 / 0 \\
8 / 21\end{array}$ & $\begin{array}{l}\text { Equipo } \\
\text { de } \\
\text { seguimie } \\
\text { nto }\end{array}$ & $\begin{array}{c}\text { No } \\
\text { comenza } \\
\text { do }\end{array}$ \\
\hline & $\begin{array}{l}\text { Plan de } \\
\text { mejora } \\
\text { continua }\end{array}$ & 20 & 4 & FC & -3 & $\begin{array}{c}31 / 07 \\
/ 21\end{array}$ & $\begin{array}{l}19 / 0 \\
8 / 21\end{array}$ & $\begin{array}{l}\text { Equipo } \\
\text { de } \\
\text { evaluació } \\
n\end{array}$ & $\begin{array}{c}\text { No } \\
\text { comenza } \\
\text { do }\end{array}$ \\
\hline
\end{tabular}

Fuente: Elaboración propia.

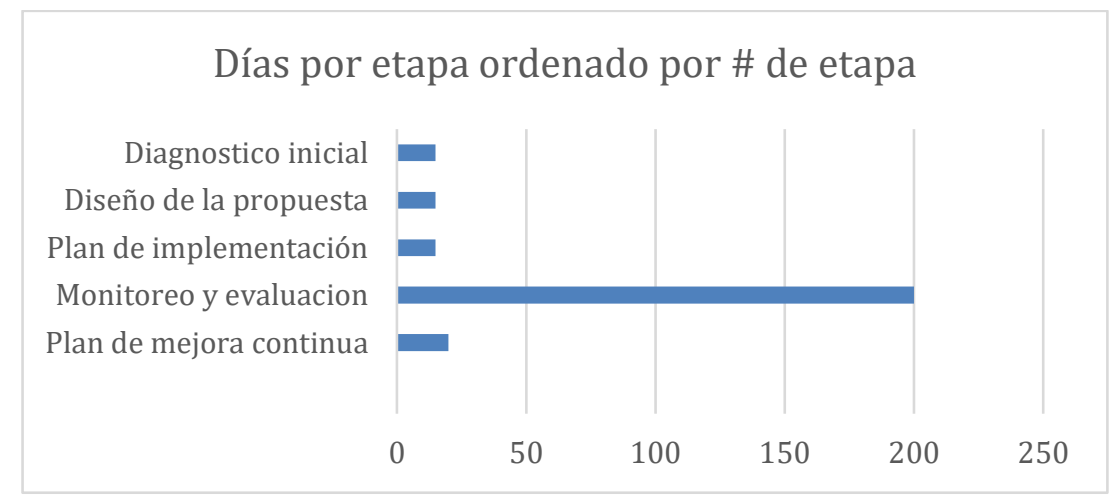

Figura 4. Días por etapa.

Fuente: Elaboración propia.

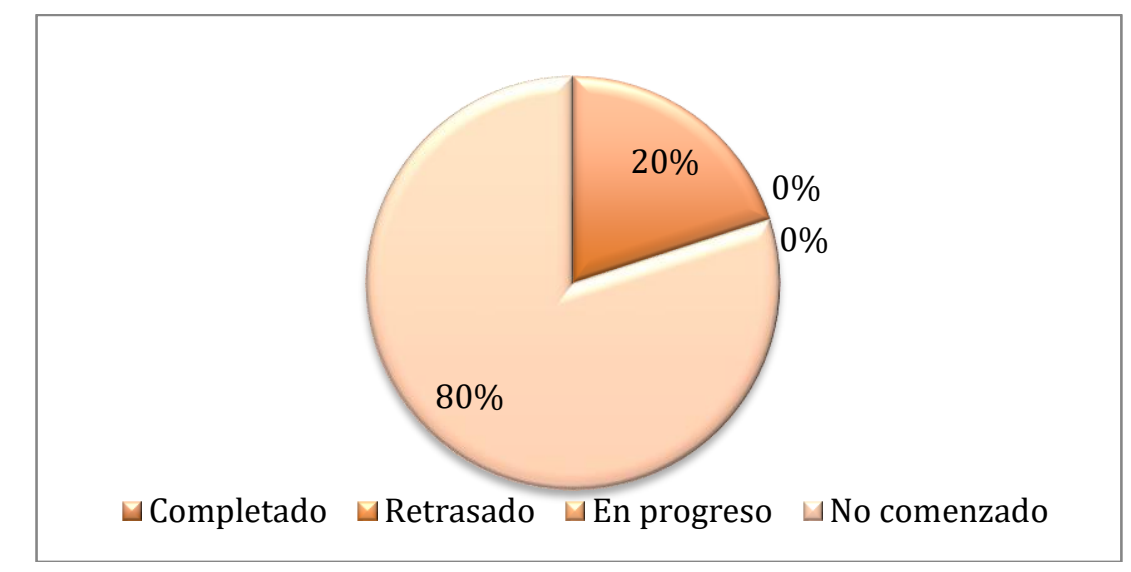

Figura 5. Estatus de cumplimiento.

Fuente: Elaboración propia. 
Revista Interdisciplinaria de Humanidades, Educación, Ciencia y Tecnología

Año VII. Vol. VII. N¹2. Enero - Junio. 2021

Hecho el depósito de ley: pp201602FA4721

ISSN-L: 2542-3029; ISSN: 2610-802X

Universidad Nacional Experimental Francisco de Miranda (UNEFM). Santa Ana de Coro. Venezuela

Milton Junior Rivadeneira-Hermida; Juan Carlos Erazo-Álvarez; Katina Vanessa Bermeo-Pazmiño

Diego Patricio Cisneros-Quintanilla

\section{CONCLUSIONES}

Se puede concluir, con base a los datos obtenidos durante la investigación, los cambios administrativos y organizacionales en CEFAS, son necesarios especialmente en las actuales condiciones de crisis sanitaria.

El modelo propuesto cumple con el objetivo planteado en la investigación que es generar cambios administrativos y organizacionales en CEFAS, que permite mejorar las condiciones laborales de los colaboradores y atención a grupos prioritarios.

La aplicación de la propuesta en mención ayuda a la dirección, la toma de decisiones tanto en el ámbito del manejo de la organización, como en la administración del capital humano, optimizando el uso de los recursos que la Prefectura de Morona Santiago asigna para la organización.

Los colaboradores están conformes con la labor de la organización y su ambiente laboral, sin embargo, es importante enfocar la gestión en un proceso de mejora continua que permita aumentar la cobertura a beneficiarios de grupos vulnerables con un ambiente laboral digno para los colaboradores.

\section{REFERENCIAS CONSULTADAS}

CEPAL. (2020). América Latina y el Caribe ante la pandemia del COVID-19 Efectos económicos y sociales [Latin America and the Caribbean in the Face of the COVID19 Pandemic Economic and Social Effects] Recuperado de https://n9.cl/hhbkk.

Erazo, J., Calle, K., \& Narváez, C. (2020). Marketing digital y estrategias online en el sector de fabricación de muebles de madera [Digital marketing and online strategies in the wood furniture manufacturing sector]. Revista Arbitrada Interdisciplinaria Koinonía, 5(10), 339-369. http://dx.doi.org/10.35381/r.k.v5i10.698

Lazo, N., Erazo, J., \& Narváez, C. (2019). El Balanced Scorecard como herramienta de control interno en el sector Manufacturero [The Balanced Scorecard as an internal control tool in the Manufacturing sector]. Revista Arbitrada Interdisciplinaria Koinonía, 4(1), 125-152. http://dx.doi.org/10.35381/r.k.v4i1.374 


\begin{abstract}
CIENCIAMATRIA
Revista Interdisciplinaria de Humanidades, Educación, Ciencia y Tecnología

Año VII. Vol. VII. N¹2. Enero - Junio. 2021

Hecho el depósito de ley: pp201602FA4721

ISSN-L: 2542-3029; ISSN: 2610-802X

Universidad Nacional Experimental Francisco de Miranda (UNEFM). Santa Ana de Coro. Venezuela

Milton Junior Rivadeneira-Hermida; Juan Carlos Erazo-Álvarez; Katina Vanessa Bermeo-Pazmiño

Diego Patricio Cisneros-Quintanilla
\end{abstract}

Microsoft Project. (2020). Predecesoras (campo de tareas). https://n9.cl/xm2k7

Ministerio de Salud de Costa Rica. (2020). Tipos de teletrabajo. Obtenido de https://n9.cl/b03rh

Ministerio de Trabajo. (2016). Acuerdo ministerial Nro. MDT-2016-190. Acuerdo ministerial Nro. MDT-2016-190. Quito, Pichincha, Ecuador: Ministerio de Trabajo.

Ministerio de Trabajo. (2017). Acuerdo Ministerial Nro. MDT-2017-0090-A. Acuerdo Ministerial Nro. MDT-2017-0090-A. Quito, Pichincha, Ecuador: Ministerio de Trabajo.

Ministerio de Trabajo. (2020). Teletrabajo. Recuperado de https://n9.cl/v37e

Mintzberg, H. (1984). Power and Organization Life Cycles. AMR, 9, 207224. https://doi.org/10.5465/amr.1984.4277632

Organización Internacional del Trabajo, OIT. (2020). OIT: EI COVID-19 causa pérdidas devastadoras de empleos y horas de trabajo. Recuperado de https://n9.cl/zx3z

Pardo-Díaz, M. P. \& Rodríguez-Martín, D. C. (2020). El teletrabajo en tiempos de COVID19. Trabajo de Grado. Universidad Católica de Colombia. Facultad de Ciencias Económicas y Administrativas. Programa de Economía. Especialización en Administración Financiera. Bogotá, Colombia. https://hdl.handle.net/10983/24640

Pérez, A. (2015). Tipos de estructuras organizativas. Cuál es la mejor para tu empresa. Recuperado de https://n9.cl/5o2n

Picatoste, F. (2020). 10 medidas que deben adoptar las empresas para afrontar una pandemia. Recuperado de https://n9.cl/gwxd

Prefectura de Morona Santiago. (2019). Quienes Somos Cefas. Recuperado de septiembre https://n9.cl/gs3ri

Presidencia de la República del Ecuador. (2010). Ley orgánica de servicio público. Ley orgánica de servicio público. Quito, Pichincha, Ecuador: Asamblea Nacional. 


\section{CIENCIAMATRIA}

Revista Interdisciplinaria de Humanidades, Educación, Ciencia y Tecnología

Año VII. Vol. VII. N¹2. Enero - Junio. 2021

Hecho el depósito de ley: pp201602FA4721

ISSN-L: 2542-3029; ISSN: 2610-802X

Universidad Nacional Experimental Francisco de Miranda (UNEFM). Santa Ana de Coro. Venezuela

Milton Junior Rivadeneira-Hermida; Juan Carlos Erazo-Álvarez; Katina Vanessa Bermeo-Pazmiño Diego Patricio Cisneros-Quintanilla

Project Management Institute. (2013). Fundamentos para la dirección de proyectos (guía del PMBOK) [Fundamentals of project management (PMBOK guide)]. Pensilvania: Project Management Institute, Inc.

(C2021 por los autores. Este artículo es de acceso abierto y distribuido según los términos y condiciones de la licencia Creative Commons Atribución-NoComercial-Compartirlgual 4.0 Internacional (CC BY-NC-SA 4.0)

(https://creativecommons.org/licenses/by-nc-sa/4.0/). 